\title{
Automutilação oral em crianças e adolescentes e fatores associados: revisão integrativa
}

Oral self-mutilation in child and adolescents and associated factors: integrative review

La automutilación oral en niños y adolescentes y factores asociados: revisión integradora

José Rodolfo Tavares de Melo

ORCID: https://orcid.org/0000-0003-3218-4580 Universidade de Pernambuco, Brasil E-mail: jrodolfo.melo@upe.br

Niviane Marielly da Costa Oliveira ORCID: https://orcid.org/0000-0002-8258-4876 Universidade de Pernambuco, Brasil E-mail: nivycosta@gmail.com

Camila de Paula Rosendo

ORCID: https://orcid.org/0000-0001-5157-2605

Universidade de Pernambuco, Brasil

E-mail: camiladpaularosendo@gmail.com

Alice Kelly Barreira

ORCID: https://orcid.org/0000-0002-0351-9022

Universidade Federal de Pernambuco, Brasil E-mail: alicekelly@yahoo.com

Viviane Colares

ORCID: https://orcid.org/0000-0003-2912-2100

Universidade de Pernambuco, Brasil

E-mail: viviane.colares@upe.br

\begin{abstract}
Resumo
Introdução: O comportamento autolesivo é definido como um ato dirigido a si mesmo que resulta em um dano tecidual. As estruturas orais podem ser traumatizadas por esse tipo de comportamento e sua incidência vem aumentando entre as crianças. Objetivo: Apresentar os fatores associados à automutilação oral em crianças e adolescentes, atentando para o diagnóstico diferencial das lesões orais. Metodologia: Foi realizada uma revisão integrativa da literatura a partir de pesquisa nas bases de dados Medline (PubMed), entre 2010 e 2020. Foram selecionados 27 artigos, sendo 5 caso-controle, 1 transversal e 21 relatos de caso. Resultados: Os estudos avaliados foram desenvolvidos em diversos países, em hospitais, ambulatórios, institutos, universidades, escolas e orfanato. Nos estudos observacionais as amostras variaram entre 30 e 303 participantes. A automutilação oral foi relacionada aos fatores sociais, hereditários, neurológicos e psiquiátricos. Observou-se também o envolvimento de indivíduos sem comorbidades em casos de automutilação oral. Nesses estudos houve mais crianças que adolescentes envolvidos em quadros de automutilação oral. Conclusão: Fatores hereditários, neurológicos, psiquiátricos e sociais são apontados como fatores associados aos quadros de automutilação oral em crianças e adolescentes. Entretanto, a literatura ainda é escassa em padronização de protocolo para diagnóstico de automutilação oral, assim outros estudos são necessários para determinação de quais fatores podem ser pertinentes a esses casos quando envolvem crianças e adolescentes.
\end{abstract}

Palavras-chave: Criança; Adolescente; Automutilação; Comportamento autolesivo.

\begin{abstract}
Introduction: Self-injurious behaviour is defined as an act directed at oneself that results in tissue damage. Oral structures can be traumatized by this type of behaviour and its incidence is increasing among children. Objective: To present the factors associated with oral self-mutilation in children and adolescents, in order to assist the clinician in the differential diagnosis of oral lesions. Methodology: An integrative literature review was carried out based on a search in the Medline databases (PubMed), between 2010 and 2020. 27 articles were selected, being 5 case-control, 1 cross-sectional and 21 case reports. Results: The evaluated studies were developed in several countries, in hospitals, outpatient clinics, institutes, universities, schools and orphanages. In observational studies, the samples varied between 30 and 303 participants. Oral self-mutilation was related to social, hereditary, neurological and psychiatric factors. It was also observed the involvement of individuals without comorbidities in cases of oral self-mutilation. In these studies, there were more children than adolescents involved in oral self-mutilation. Conclusion: Hereditary, neurological, psychiatric and social factors are pointed out as factors associated with oral self-mutilation in children
\end{abstract}


and adolescents. However, the literature is still scarce in standardizing the protocol for diagnosing oral self-mutilation, so further studies are needed to determine what factors may be relevant to these cases when involving children and adolescents.

Keywords: Child; Teenager; Self-mutilation; Self-injurious behaviour.

\section{Resumen}

Introducción: La conducta autolesiva se define como un acto dirigido a uno mismo que resulta en daño tisular. Las estructuras orales pueden resultar traumatizadas por este tipo de comportamiento y su incidencia está aumentando entre los niños. Objetivo: Presentar los factores asociados a la automutilación oral en niños y adolescentes, prestando atención al diagnóstico diferencial de las lesiones bucales. Metodología: Se realizó una revisión integradora de la literatura a partir de una búsqueda en las bases de datos de Medline (PubMed), entre 2010 y 2020.Se seleccionaron 27 artículos, siendo 5 caso-control, 1 transversal y 21 casos clínicos. Resultados: Los estudios evaluados se desarrollaron en varios países, en hospitales, ambulatorios, institutos, universidades, escuelas y orfanatos. En los estudios observacionales, las muestras variaron entre 30 y 303 participantes. La automutilación oral se relacionó con factores sociales, hereditarios, neurológicos y psiquiátricos. También se observó la participación de personas sin comorbilidades en los casos de automutilación oral. En estos estudios, hubo más niños que adolescentes involucrados en la automutilación oral. Conclusión: Se señalan factores hereditarios, neurológicos, psiquiátricos y sociales como factores asociados a la automutilación oral en niños y adolescentes. Sin embargo, la literatura aún es escasa para estandarizar el protocolo de diagnóstico de la automutilación oral, por lo que se necesitan más estudios para determinar qué factores pueden ser relevantes en estos casos cuando se trata de niños y adolescentes.

Palabras clave: Niño; Adolescente; Automutilación; Comportamiento autolesivo.

\section{Introdução}

O comportamento autolesivo, do inglês Self-Injurious Behaviors (SIB) foi definido como um ato dirigido a si mesmo que resulta em um dano tecidual (Shapira et al., 2016; Tate \& Baroff, 1966). Ele também é referido na literatura como lesões automutilantes, hábitos masoquistas e lesões factícias. Geralmente os quadros de automutilação se manifestam como pancadas no corpo ou na cabeça, mordidas de dedos e lábios, cortes na pele, automutilação oral, ocular e genital (Mistry et al., 2010; Subbaiah, Thomas, 2015).

É interessante destacar que as estruturas orais e periorais podem ser traumatizadas por esse tipo de comportamento e quando esse afeta essas estruturas envolve mordeduras no lábio, mucosa jugal (Chen \& Liu, 1996; Romero et al., 2005) superfícies laterais da língua (Chen \& Liu, 1996; Flaitz \& Felefli, 2000) e autoextração dentária (Williams, 2015). A lesão ocorre principalmente pela introdução de objetos estranhos ou unhas na cavidade oral ou pela mordida dos tecidos orais. Desse modo, se apresentam como úlceras factícias, gengivite facticial, periodontite facticial e autoextração (Medina et al., 2003; N. Mistry et al., 2017), e a depender de sua frequência e gravidade, o SIB pode gerar vários graus de autoferimentos (Romero et al., 2005).

Em aspectos epidêmicos, a autolesão pode afetar indivíduos de qualquer idade ou sexo e sua incidência vem aumentando entre as crianças (Kwon et al., 2015; Limeres et al., 2013). É importante enfatizar que mais da metade das lesões facticiais estão localizadas na região de cabeça e pescoço (Medina et al., 2003; Mistry et al., 2017).

No que concerne à possível etiologia da automutilação, pode-se de uma maneira ampla classificá-la em duas categorias: orgânica e funcional (Ayer \& Levin, 1974; Hildebrand, Carvalho, da Rosa, Martins, \& Sant'Ana Filho, 2011). Orgânica quando os ferimentos são infligidos inconscientemente, não intencionalmente e compulsivamente (Altom \& DiAngelis, 1989; Ayer \& Levin, 1974; Hildebrand, Carvalho, da Rosa, Martins, \& Sant'Ana Filho, 2011) e funcional nos casos em que a autolesão é realizada sabidamente por paciente sem comorbidades associadas (Hildebrand, Carvalho, da Rosa, Martins, \& Sant'Ana Filho, 2011; Stewart \& Kernohan, 1973).

Sendo assim, o objetivo deste estudo é apresentar, com base na literatura, quais fatores podem estar associados à automutilação oral em crianças e adolescentes, a fim de ajudar o clínico no diagnóstico diferencial das lesões orais. 


\section{Metodologia}

Foi realizada uma revisão integrativa da literatura. Trata-se de um estudo cujo a coleta de dados foi realizada a partir de fontes secundárias, por meio de levantamento bibliográfico (Pereira et al., 2018). A revisão integrativa foi construída através de seis etapas: 1. Elaboração da pergunta norteadora; 2. Busca na literatura; 3. Seleção dos trabalhos que compuseram a amostra da revisão; 4. Análise crítica dos estudos incluídos; 5. Síntese e discussão dos resultados; 6. Apresentação da revisão, com consequente exame crítico dos resultados.

A questão norteadora elaborada foi: Quais fatores estão associados a automutilação oral em crianças e adolescentes? Utilizou-se a estratégia PECO, acrônimo no idioma inglês e que em português corresponde aos seguintes elementos: P população: criança e adolescente; E - exposição: automutilação, comportamento autolesivo; C - comparação, que neste caso não se aplica; $\mathrm{O}$ - desfecho: injúria oral.

Foi realizada uma busca na base de dados do portal PubMed (National Library of Medicine National Institutes of Health), inicialmente sem delimitação temporal, por um único pesquisador para artigos publicados até agosto de 2020 . Os descritores MeSH Database e termos usados foram: child, adolescent, self mutilation, self-injurious behavior, oral injury. Para a sistematização das buscas, os descritores precisaram ser entrecruzados, usando os operadores booleanos AND e OR. Assim, foram identificados inicialmente 318 artigos a partir da estratégia de busca elaborada. Após aplicação dos filtros idioma: Português, Inglês e Espanhol e intervalo de ano de publicação: últimos 10 anos, bem como removendo as duplicatas, restaram 92 estudos para leitura de títulos e resumos. Ao fim da leitura desses e pela concordância dos pesquisadores, houve a seleção de apenas 32 estudos para leitura de texto completo, por preencheram os critérios de inclusão: estar em consonância com a temática abordada; englobar a população crianças e adolescentes; e, serem estudos observacionais e clínicos. Desses 32, pela avaliação de elegibilidade, 27 artigos foram incluídos, porque os demais ou não responderam à pergunta condutora, ou não se tratava de desenhos de estudos relevantes a essa revisão, ou englobaram adultos na amostra. Desse modo, na revisão integrativa, foram incluídos 27 artigos.

Os critérios de inclusão para a seleção de estudos foram: estudos clínicos e observacionais, publicados nos idiomas inglês, português e espanhol e cujos participantes fossem crianças e adolescentes. Como critérios de exclusão foram definidos: estudos de revisão da literatura, carta ao editor, monografia, estudos que não apresentavam como desfecho automutilação oral. A seleção dos artigos foi realizada por dois pesquisadores independentes e um terceiro avaliador foi convidado nos casos de discordância entre os dois avaliadores.

Nesta pesquisa, utilizou-se o instrumento Preferred Reporting Items for Systematic Review and Meta-Analyses (PRISMA) (Moher, Liberati, Tetzlaff, 2009) para apresentar a busca e a seleção dos estudos, conforme fluxograma detalhado na Figura 1. Os artigos foram classificados em relação ao Nível de Evidência (NE), segundo a adaptação da classificação proposta por (Souza, Silva, 2010). Nível 1: meta-análise de múltiplos ensaios clínicos controlados randomizados; Nível 2: evidências de estudos individuais com design experimental; Nível 3: evidências de estudos quase experimentais; Nível 4: evidência de estudos descritivo (não experimental) ou com abordagem qualitativa; Nível 5: evidências de relatos de caso; Nível 6: evidências baseadas em opiniões de especialistas. 
Figura 1: Fluxograma da pesquisa: identificação, triagem, elegibilidade e inclusão dos artigos científicos na revisão integrativa, conforme Preferred Reporting Items for Systematic Review and Meta-Analyses (PRISMA, 2009).

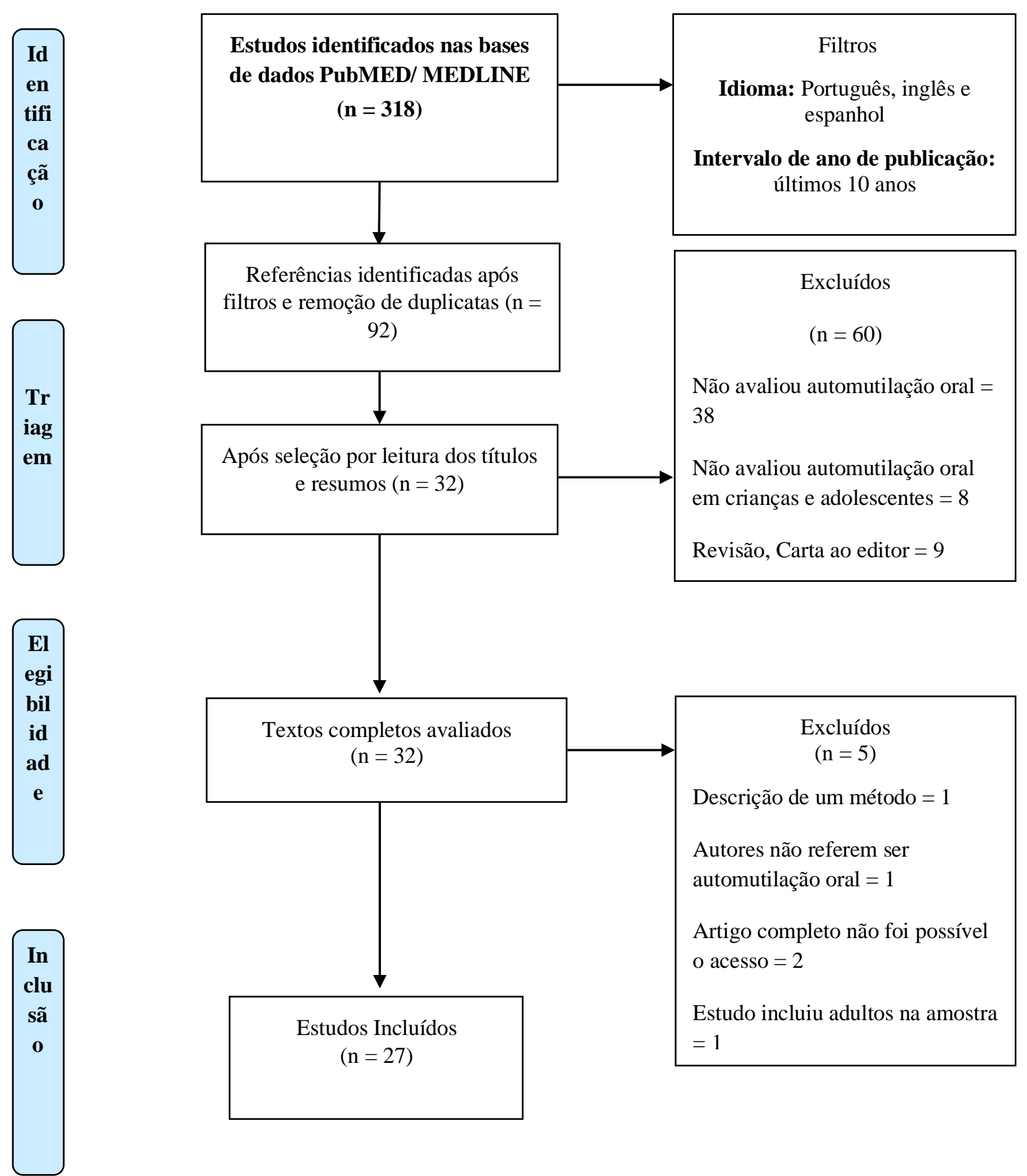

Fonte: Autores.

Posteriormente, fez-se a análise crítica e detalhada à luz dos conhecimentos teóricos à cerca do tema automutilação oral em crianças e adolescentes. Os dados dos 27 artigos selecionados para essa revisão integrativa, são apresentados em três tabelas para a apresentação dos resultados e posterior discussão, a fim de integrá-los para a construção de uma concepção geral do tema. Assim, a primeira tabela detalha a caracterização dos estudos selecionados; a segunda, descreve a síntese dos resultados dos estudos observacionais; a terceira e última tabela, refere-se aos possíveis fatores associados à automutilação oral 
em crianças e adolescentes.

\section{Resultados}

É interessante destacar que em relação às suas caracterizações, os estudos apresentam distinção no que tange à localização de execução, aos participantes e ao desenho metodológico. Eles foram desenvolvidos em diversos países e realizados em diferentes ambientes: hospitais, ambulatórios, institutos, universidades, escolas e orfanato (Tabela 1).

Tabela 1: Caracterização de produção científica sobre automutilação oral em crianças e adolescentes.

\begin{tabular}{|c|c|c|c|}
\hline Autor/ Ano & Pais & Local & Desenho do estudo (n) \\
\hline Navya et al. 2019 & Índia & Hospital & Relato de Caso (1) \\
\hline Soussou et al., 2019 & Canadá & Hospital & Relato de Caso (1) \\
\hline Suhaib et al., 2017 & Paquistão & Instituto & Caso-controle (85) \\
\hline Siragusa et al., 2013 & Itália & Instituto & Transversal (245) \\
\hline Elhennawy et al., 2017 & Alemanha & Hospital & Relato de Caso (1) \\
\hline Ashwin et al., 2015 & Índia & Hospital & Série de Casos (8) \\
\hline AlSadhan et al., 2016 & Arábia Saudita & Orfanato & Caso-controle (180) \\
\hline Olczak-Kowalczyk et al., 2011 & Polônia & Clínica Escola & Caso-controle (30) \\
\hline Panek et al., 2012 & Polônia & Escola Técnica & Transversal (303) \\
\hline Arhakis et al., 2010 & Grécia & Clínica Escola & Relato de Caso (1) \\
\hline Wadenya et al., 2011 & EUA & Hospital & Relato de Caso (1) \\
\hline La Cuadra et al., 2016 & Espanha & Hospital & Relato de Caso (2) \\
\hline Fantuzzo et al., 2014 & EUA & Instituto de Odontopediatria & Relato de Caso (1) \\
\hline Mowafy, Wahba, Sharaf, 2017 & Egito & Clínica Escola & Relato de Caso (1) \\
\hline El Khatib et al., 2013 & Egito & Instituições e escolas & Caso-controle (200) \\
\hline Rangeeth et al., 2011 & Índia & Ambulatório & Relato de Caso (1) \\
\hline Kwon et al., 2015 & Coreia do Sul & Hospital & Relato de Caso (1) \\
\hline Gaur et al., 2018 & Índia & Ambulatório & Relato de Caso (1) \\
\hline Brissaud et al., 2020 & França & Hospital & Relato de Caso (1) \\
\hline Hildebrand et al., 2011 & Brasil & Clínica Escola & Relato de Caso (1) \\
\hline Gantha et al., 2017 & Índia & Clínica Escola & Relato de Caso (1) \\
\hline Gao et al., 2013 & China & Clínica Escola & Relato de Caso (1) \\
\hline Williams, 2015 & Reino Unido & Clínica Escola & Relato de Caso (1) \\
\hline Romero et al., 2014 & Espanha & Hospital & Relato de Caso (1) \\
\hline Autor/ Ano & Pais & Local & Desenho do estudo (n) \\
\hline Herrera, Pollock, 2014 & Colômbia & Hospital & Relato de Caso (1) \\
\hline Mistry et al., 2017 & Índia & Clínica Escola & Relato de Caso (1) \\
\hline Labib et al., 2011 & Marrocos & Hospital & Relato de Caso (2) \\
\hline
\end{tabular}

Fonte: Autores. 
Research, Society and Development, v. 10, n. 2, e29710212493, 2021

(CC BY 4.0) | ISSN 2525-3409 | DOI: http://dx.doi.org/10.33448/rsd-v10i2.12493

No que concerne ao delineamento metodológico, vinte e um estudos foram relatos de caso (NE 5), quatro casocontrole (NE 3), e um transversal (NE 4). Com relação ao tamanho da amostra, quando dos estudos observacionais, observouse variação de 30 a 303 participantes.

A Tabela 2 descreve a síntese dos resultados dos estudos observacionais (caso-controle e transversal). Destaca-se que a maior prevalência de automutilação oral está relacionada a fatores sociais, hereditários, neurológicos e psiquiátricos. Todavia, há envolvimento também de indivíduos sem comorbidades em casos de automutilação oral. É evidente nesses estudos que mais crianças que adolescentes foram mais envolvidas em quadros de automutilação oral (AlSadhan \& Al-Jobair, 2017a; Ashwin et al., 2015; Fantuzzo et al., 2014; Soussou et al., 2019; Suhaib et al., 2019). 
Research, Society and Development, v. 10, n. 2, e29710212493, 2021

(CC BY 4.0) | ISSN 2525-3409 | DOI: http://dx.doi.org/10.33448/rsd-v10i2.12493

Tabela 2: Síntese dos principais resultados dos estudos observacionais.

\begin{tabular}{|c|c|c|c|c|}
\hline Artigo & Desenho Estudo & Amostra/ População & Sexo & Conclusão \\
\hline $\begin{array}{l}\text { Suhaib et al., } \\
2017\end{array}$ & Caso-controle. & $\begin{array}{l}85 \text { crianças, entre } 2 \text { e } 10 \\
\text { anos de idade, } 57 \text { com TEA } \\
\text { e } 27 \text { controles. }\end{array}$ & $\begin{array}{l}\text { Masculino/ } \\
\text { Feminino }\end{array}$ & $\begin{array}{l}\text { Nenhum comportamento autoinfligido foi relatado pelos pais de crianças com TEA. } \\
\text { No entanto, } 3 \% \text { das crianças apresentaram úlceras labiais. }\end{array}$ \\
\hline $\begin{array}{l}\text { Siragusa et al., } \\
2013\end{array}$ & Transversal. & $\begin{array}{l}245 \text { crianças e adolescentes } \\
\text { com retardo mental entre } 3 \\
\text { e } 14 \text { anos de idade. }\end{array}$ & $\begin{array}{l}\text { Masculino/ } \\
\text { Feminino }\end{array}$ & $\begin{array}{l}\text { As lesões de automutilação da boca são muito frequentes em indivíduos com retardo } \\
\text { mental. Nesse estudo } 65,8 \% \text { apresentaram lesão em língua; } 60,3 \% \text { em lábio; e } 41,4 \% \\
\text { em mucosa bucal. }\end{array}$ \\
\hline $\begin{array}{l}\text { AlSadhan et } \\
\text { al., } 2016\end{array}$ & $\begin{array}{l}\text { Transversal caso- } \\
\text { controle. }\end{array}$ & $\begin{array}{l}180 \text { crianças (90 órfãs e } 90 \\
\text { não órfãs) entre } 4 \text { e } 12 \\
\text { anos. }\end{array}$ & $\begin{array}{l}77 \% \\
\text { masculino e } \\
33 \% \\
\text { feminino. }\end{array}$ & $\begin{array}{l}\text { Das } 25 \text { crianças órfãs }<6 \text { anos, } 1 \text { apresentou automutilação oral; entre } 7-9 \text { anos }(31), 3 \\
\text { apresentaram automutilação oral; }>10 \text { anos }(34), 4 \text { apresentaram automutilação oral. } \\
\text { As crianças do grupo controle nessa faixa etária não apresentaram automutilação oral. } \\
\text { As crianças órfãs tinham mais hábitos de sucção digital }(58,9 \%) \text { e automutilação oral } \\
(8,9 \%) \text { do que as crianças controle e essa diferença foi estatisticamente significativa } \\
(p<0,001 \text { e } p=0,003 \text {, respectivamente). }\end{array}$ \\
\hline $\begin{array}{l}\text { Olczak- } \\
\text { Kowalczyk et } \\
\text { al., } 2011\end{array}$ & Caso-controle & $\begin{array}{l}30 \text { crianças e adolescentes } \\
(15 \text { crianças com PWS - } \\
\text { idade média de } 9,8 \pm 4,4 \\
\text { anos - e } 15 \text { crianças } \\
\text { saudáveis - idade média de } \\
11,5 \pm 3,5 \text { anos). }\end{array}$ & $\begin{array}{l}\text { Masculino/ } \\
\text { Feminino }\end{array}$ & $\begin{array}{l}\text { Os autores concluem que, as lesões de automutilação oral foram encontradas em } 8 \text { das } \\
15 \text { crianças e adolescentes. Elas estavam associadas a mordidas nas bochechas e } \\
\text { lábios. }\end{array}$ \\
\hline $\begin{array}{l}\text { Panek et al., } \\
2012\end{array}$ & Transversal & $\begin{array}{l}303 \text { adolescentes saudáveis, } \\
\text { idade média de } 18,8 \text { anos. }\end{array}$ & $\begin{array}{l}\text { Masculino/ } \\
\text { Feminino }\end{array}$ & $\begin{array}{l}\text { Os autores concluíram que quase todos os sujeitos revelaram morder a mucosa do } \\
\text { lábio ou bochecha. }\end{array}$ \\
\hline $\begin{array}{l}\text { El khatib et al., } \\
2013\end{array}$ & Caso-controle & $\begin{array}{l}200 \text { crianças e adolescentes } \\
\text { entre } 3 \text { e } 13 \text { anos ( } 100 \text { com } \\
\text { TEA e } 100 \text { saudáveis). }\end{array}$ & $\begin{array}{l}\text { Masculino/ } \\
\text { Feminino }\end{array}$ & $\begin{array}{l}\text { Ao avaliar automutilação oral, os pais de crianças com TEA relataram uma maior } \\
\text { prevalência de comportamentos autolesivos do que pais de crianças saudáveis DP } \\
<0,0001) \text {. Comportamento autolesivo e bruxismo foram mais praticados por crianças } \\
\text { com TEA ( } 32 \% \text { das crianças com TEA e } 2 \% \text { das crianças saudáveis, } \mathrm{P}<0,001 \text { ). } \\
\text { Comportamentos autolesivos foram mais praticados por crianças com TEA do que } \\
\text { por crianças saudáveis. }\end{array}$ \\
\hline
\end{tabular}

Fonte: Autores. 
Na Tabela 3 são apontados através da associação entre automutilação oral e condições específicas, os quadros nos quais essa condição frequentemente é relatada na literatura. Os quadros mais identificados nesta revisão foram os que se relacionam aos fatores neurológicos, psiquiátricos, hereditários e sociais. Aparecendo com maior destaque os fatores hereditários associados a eventos de automutilação oral. É importante destacar que em três estudos incluídos pacientes sem comorbidades apresentaram automutilação oral.

Tabela 3: Fatores associados a automutilação oral em crianças e adolescentes apontados pelos estudos.

\begin{tabular}{l|l}
\hline Fatores & Artigos \\
\hline Neurológicos & $\begin{array}{l}\text { Leucoencefalopatia megalencefálica - MLC (Fantuzzo et al., 2014); Doença de Santovuori- } \\
\text { Haltia-Hagberg (Lipofuscinoses Ceriod Neuronal Infantil) (Mistry et al., 2017); Distonia } \\
\text { (Brissaud et al., 2020). }\end{array}$ \\
\hline Psiquiátricos & $\begin{array}{l}\text { Transtorno psiquiátrico (Herrera, Pollock, 2014); Transtorno do Espectro Autista - TEA } \\
\text { (Suhaib et al., 2017; El Khatib et al., 2013; Gao et al., 2013). }\end{array}$ \\
\hline Hereditários & $\begin{array}{l}\text { Insensibilidade congênita à dor (Navya et al. 2019; Soussou et al., 2019; Elhennawy et al., } \\
\text { 2017; Ashwin et al., 2015; Gaur et al., 2018; Gao et al., 2013; Labib et al., 2011); Síndrome de } \\
\text { Prader-Willi - PWS (Olczak-Kowalczyk et al., 2011); Sindrome de Lesch-Nyhan (Arhakis et } \\
\text { al., 2010; la Cuadra et al., 2016; Romero Maroto et al., 2014); Síndrome de Kabuki (Wadenya } \\
\text { et al., 2011); Síndrome de Joubert Plus (Mowafy, Wahba, Sharaf, 2017). }\end{array}$ \\
\hline Fatores sociais & Violência (Hildebrand et al., 2011; Gantha et al., 2017): Orfandade (AlSadhan et al., 2016). \\
\hline Sem comorbidades & (Panek et al., 2012; Rangeeth et al., 201; Kwon et al., 2015). \\
\hline
\end{tabular}

Fonte: Autores.

De acordo com os artigos considerados, o sexo masculino apareceu isolado em 15 estudos, (Arhakis et al., 2010; Elhennawy et al., 2017; Fantuzzo et al., 2014; Gantha et al., 2017; Gao et al., 2013; Gaur et al., 2018; Gisbert de la Cuadra et al., 2016a; Kwon et al., 2015; Mowafy et al., 2017b; Navya et al., 2019; Rangeeth et al., 2011; Romero Maroto et al., 2014; Soussou et al., 2019; Wadenya et al., 2011; Williamson et al., 2016); o feminino isolado em 4 estudos (Brissaud et al., 2016; Herrera \& Pollock, 2014; Hildebrand, Carvalho, da Rosa, Martins, \& Filho, 2011; Mistry et al., 2017) e ambos os sexos em 8 estudos (Ashwin et al., 2015; El Khatib et al., 2014; Labib et al., 2011; Olczak-Kowalczyk et al., 2011; Panek et al., 2012; Siragusa et al., 2013; Suhaib et al., 2017). Quando observada a população mais frequentemente acometida por automutilação oral, o grupo "crianças" apareceu em 21 estudos (AlSadhan \& Al-Jobair, 2017b; Ashwin et al., 2015; Brissaud et al., 2016; El Khatib et al., 2013; Fantuzzo et al., 2014; Gao et al., 2013; Gaur et al., 2018; Gisbert de la Cuadra et al., 2016b; Hildebrand, Carvalho, da Rosa, Martins, \& Filho, 2011; Kwon et al., 2015; Labib et al., 2011; L. N. Mistry et al., 2017; Mowafy et al., 2017a; Navya et al., 2019; Olczak-Kowalczyk et al., 2011; Romero Maroto et al., 2014; Siragusa et al., 2013; Soussou et al., 2019; Suhaib et al., 2017; Wadenya et al., 2011; Williams, 2015), enquanto que os "adolescentes", em 11 estudos (Ashwin et al., 2015; Carra et al., 2013; Elhennawy et al., 2017; Gantha et al., 2017; Gisbert de la Cuadra et al., 2016b; Herrera \& Pollock, 2014; Olczak-Kowalczyk et al., 2011; Panek et al., 2012; Rangeeth et al., 2011; Siragusa et al., 2013; Topouzelis et al., 2010). 


\section{Discussão}

A etiologia de uma lesão oral por automutilação pode ser diversa e diagnósticos diferenciais devem serem feitos entre esta condição e outros comportamentos (Hildebrand, Carvalho, da Rosa, Martins, \& Sant'Ana Filho, 2011). De acordo com (Kwon et al., 2015), existem diversos fatores etiológicos que contribuem para a mordedura na língua, entre esses: deficiência de desenvolvimento, que pode estar associada a uma síndrome como Lesch-Nyhan, neuropatia causando insensibilidade à dor ou mesmo indivíduos sem história médica relevante . Nesta revisão estes fatores etiológicos foram encontrados em casos de automutilação oral (Tabela 3).

De acordo com os estudos realizados por alguns autores (Ayer \& Levin, 1974; Hildebrand, Carvalho, da Rosa, Martins, \& Filho, 2011; Rodd, 1995; Stewart \& Kernohan, 1973), a automutilação oral foi mais comumente observada em crianças do que em adolescentes e adultos. Observou-se nesse trabalho, que dos 27 estudos selecionados, 21 foram realizados com crianças. Porém, observa-se que a maior parte dos estudos coletados evidenciou análises em crianças.

Quando a automutilação é observada em crianças pequenas, um componente psicossocial está frequentemente presente, por exemplo, ansiedade, necessidade de atenção ou um sentimento de abandono (Ayer \& Levin, 1974; Stewart \& Kernohan, 1972). A esse respeito, a subsistência em um orfanato, por exemplo, apesar de oferecer abrigo, alimentos e segurança física, pode não oferecer segurança psicológica. Como efeito, crianças órfãs são uma população de risco para desenvolvimento psicossocial atípico (Ahmad et al., 2005). No estudo caso-controle realizado com 180 crianças, descobriu-se que as crianças órfãs tinham mais hábitos de automutilação oral, 8,9\% do que as crianças controle, saudáveis. Cerca de 9\% dos órfãos pesquisados neste estudo apresentaram problemas psicológicos, que podem estar relacionados ao hábito de automutilação. A presença de automutilação oral entre crianças órfãs que desconhecem seus pais é um indicador alarmante de transtornos psicológicos, nesse mesmo estudo, esse indicador aumentou de 4\% em órfãos de 6 anos e mais jovens para 11,3\% nos órfãos de 10 anos e mais velhos (AlSadhan \& Al-Jobair, 2017b). Adicionalmente, no estudo de Hildebrand et al. em 2011, a automutilação oral também foi observada no caso relatado de uma criança pela ausência dos pais, os autores chegaram à conclusão de que a ausência da mãe na rotina da criança pode ter sido desencadeadora desse comportamento autolesivo oral.

A automutilação oral também pode ser o primeiro sinal de um transtorno psiquiátrico (Cannavale et al., 2015). Mordidas crônicas de bochecha e lábios, juntamente com outras práticas de automutilação, são transtornos observados em pacientes com retardo mental, tendo sido relatado em aproximadamente 2\% deles (Butterworth \& Strean, 1963). Siragusa et al. 2013 em um estudo observacional com 245 crianças e adolescentes institucionalizados com retardo mental puderam confirmar que as lesões de automutilação da boca, como mordidas na bochecha e língua e lesões nodulares traumáticas, são muito frequentes em indivíduos com retardo mental. Do total de sua amostra, a maioria $(65,8 \%)$ apresentava algum tipo de alteração na língua; aproximadamente $60 \%$ foram afetados por alterações labiais, principalmente por sinais de mordedura e queilite; as alterações na mucosa oral também foram frequentes $(41,4 \%)$.

Quando esse transtorno psiquiátrico, automutilação, está associado a um quadro de Transtorno do Espectro Autista (TEA), as crianças apresentam graves distúrbios comportamentais. Nestes casos o comportamento é desencadeado por estresse, estímulos ou excitação e pode variar de comportamento leve, como auto-beliscões e arranhões a grave, como auto-mordidas ou bater a cabeça (Malaga et al., 2016). Em um estudo transversal na Arábia Saudita com 20 crianças com TEA, os autores verificaram que 30\% das crianças tinham lesões na região da cabeça (Murshid, 2005).

Em contrapartida, no estudo caso-controle realizado com 200 crianças e adolescentes (100 TEA e 100 controles), em instituições e escolas egípcias, foi possível comprovar que as crianças com TEA apresentam mais aspectos de desgaste dental, como uma manifestação de bruxismo, do que crianças saudáveis (El Khatib et al., 2013). Em outro estudo, realizado com 85 crianças do Paquistão, 57 com TEA e 27 controles, a presença de lesões orais compatíveis com automutilação foi verificada em 
3\% das crianças com TEA através de úlceras labiais, apesar dos pais terem negado comportamento autolesivo oral (Suhaib et al., 2017). Isso pode ter ocorrido porque recusar o diagnóstico de automutilação é uma reação comum entre os familiares (Hildebrand, Carvalho, da Rosa, Martins, \& Filho, 2011).

Além disso, no diagnóstico diferencial para comportamento autolesivo deve-se incluir insensibilidade congênita à dor, paralisia cerebral, deficiência intelectual grave, síndrome de Lesch-Nyhan (SLN), síndrome Cornélia de Lange e síndrome de Tourette (Soussou et al., 2019). Fatores hereditários precisam ser considerados, bem como outras síndromes, vista a grande incidência de automutilação sobretudo oral nestes casos. Nesse estudo, observamos que dos 27 estudos incluídos na pesquisa, 13 apresentaram como fator associado a automutilação oral uma síndrome hereditária (Tabela 3). Também foi descrito que comportamentos autodestrutivos em crianças com Síndrome Prader-Willi (SPW) podem se manifestar não apenas com automutilação na pele, mas também na mucosa oral. Em um estudo realizado com 15 crianças e adolescentes com SPW comparadas a 15 crianças e adolescentes saudáveis realizado na Polônia observou que as lesões de automutilação oral foram encontradas em 8 das 15 (bochechas 4, lábios e bochechas 1, lábios 3) crianças com SPW, enquanto 1 caso (mucosa bucal) foi encontrado no grupo saudável (controle) lábios (Olczak-Kowalczyk et al., 2019).

Em outro trabalho realizado em um hospital na Espanha, foi relatado o caso de uma criança e um adolescente com diagnóstico genético para (SLN). Em ambos os casos havia automutilação continua da língua, dos lábios e mucosa bucal não evitada por protetores bucais. Os pacientes foram submetidos a exodontia total para melhorar a qualidade de vida (Gisbert de la Cuadra et al., 2016a).

Entretanto, apesar de comumente ser observada em pacientes com alguns transtornos psiquiátricos, como transtorno de personalidade limítrofe, depressão e esquizofrenia, a automutilação tem sido relatada em pacientes sem comorbidades associadas (Rangeeth et al., 2011), a fim de determinar a prevalência e o conhecimento de determinados tipos de parafunções orais em jovens estudantes saudáveis e qualquer associação com desordem temporomandibular, selecionaram 303 adolescentes da Polônia. Ao fim, os autores verificaram que quase todos os adolescentes revelaram, entre outras, morder a mucosa do lábio ou bochecha e bruxismo (Panek et al., 2012). Know et al 2013. também relataram o caso de um menino de 7 anos com histórico de lesão ulcerativa na língua lateral há quatro semanas e sem história médica relevante. Eles concluíram que paciente neste caso tinha trauma oral autoinfligido causado, provavelmente pela posição próxima aos dentes permanentes recentemente irrompidos.

\section{Conclusão}

Através dos estudos selecionados pôde-se concluir que as lesões de automutilação foram mais observadas em crianças, entretanto esses estudos abordaram sua frequência também em adolescentes. Geralmente um fator hereditário está pertinente aos casos de automutilação oral, todavia fatores de ordem neurológica, psiquiátrica e sociais documentados podem estar associados a automutilação oral em crianças e adolescentes. A literatura ainda é escassa em padronização de protocolo para diagnóstico de automutilação oral, estudos observacionais e relatos de casos refletem esse comportamento em casos específicos, todavia carece de evidências científicas. Sendo assim, mais estudos do tipo ensaios clínicos randomizados e estudos observacionais são necessários para determinação real de quais fatores podem estar relacionados aos casos de automutilação com repercussão oral em crianças e adolescentes.

\section{Referências}

Ahmad, A., Qahar, J., Siddiq, A., Majeed, A., Rasheed, J., Jabar, F., \& von Knorring, A.-L. (2005). A 2-year follow-up of orphans' competence, socioemotional problems and post-traumatic stress symptoms in traditional foster care and orphanages in Iraqi Kurdistan. Child: Care, Health and Development, 31(2), 203-215. https://doi.org/10.1111/j.1365-2214.2004.00477.x 
AlSadhan, S. A., \& Al-Jobair, A. M. (2017a). Oral habits, dental trauma, and occlusal characteristics among 4- to 12-year-old institutionalized orphan children in Riyadh, Saudi Arabia. Spec Care Dentist, 37(1), 10-18. http://dx.doi.org/10.1111/scd.12187

AlSadhan, S. A., \& Al-Jobair, A. M. (2017b). Oral habits, dental trauma, and occlusal characteristics among 4- to 12-year-old institutionalized orphan children in Riyadh, Saudi Arabia. Special Care in Dentistry, 37(1), 10-18. https://doi.org/10.1111/scd.12187

Altom, R. L., \& DiAngelis, A. J. (1989). Multiple autoextractions: Oral self-mutilation reviewed. Oral Surgery, Oral Medicine, Oral Pathology, 67(3), 271274. https://doi.org/10.1016/0030-4220(89)90352-6

Arhakis, A., Topouzelis, N., Kotsiomiti, E., \& Kotsanos, N. (2010). Effective treatment of self-injurious oral trauma in Lesch-Nyhan syndrome: A case report. Dental Traumatology, 26(6), 496-500. https://doi.org/10.1111/j.1600-9657.2010.00930.x

Ashwin, D. P., Chandan, G. D., Jasleen, H. K., Rajkumar, G. C., Rudresh, K. B., \& Prashanth, R. (2015). Hereditary sensory and autosomal peripheral neuropathy-type IV: case series and review of literature. Oral and Maxillofacial Surgery, 19(2), 117-123. https://doi.org/10.1007/s10006-015-0486-5

Ayer, W. A., \& Levin, M. P. (1974). Self-mutilating behaviors involving the oral cavity. Journal of Oral Medicine, 29(1), 4-7.

Brissaud, O., Thébaud, N.-B., Guichoux, J., Smirani, R., Villega, F., \& Devillard, R. (2016). Case Report of a Severe Recurrent Tongue Self-Injury in an Infant With Dystonia. Pediatrics, 138(5). https://doi.org/10.1542/peds.2016-0738

Butterworth, T., \& Strean, L. P. (1963). Behavior disorders of interest to dermatologists. Archives of Dermatology, 88, 859-867. https://doi.org/10.1001/archderm.1963.01590240183031

Cannavale, R., Itro, A., Campisi, G., Compilato, D., \& Colella, G. (2015). Oral self-injuries: clinical findings in a series of 19 patients. Medicina Oral, Patologia Oral y Cirugia Bucal, 20(2), e123-9. https://doi.org/10.4317/medoral.19643

Carra, M. C., Huynh, N. T., El-Khatib, H., Remise, C., \& Lavigne, G. J. (2013). Sleep bruxism, snoring, and headaches in adolescents: short-term effects of a mandibular advancement appliance. Sleep Med, 14(7), 656-661. https://dx.doi.org/10.1016/j.sleep.2013.03.009

Chen, L. R., \& Liu, J. F. (1996). Successful treatment of self-inflicted oral mutilation using an acrylic splint retained by a head gear. Pediatric Dentistry, $18(5), 408-410$.

El Khatib, A. A., El Tekeya, M. M., El Tantawi, M. A., \& Omar, T. (2013). Oral health status and behaviours of children with Autism Spectrum Disorder: A case-control study. International Journal of Paediatric Dentistry, 24(4), 314-323. https://doi.org/10.1111/ipd.12067

El Khatib, A. A., El Tekeya, M. M., El Tantawi, M. A., \& Omar, T. (2014). Oral health status and behaviours of children with Autism Spectrum Disorder: a case-control study. International Journal of Paediatric Dentistry, 24(4), 314-323. https://doi.org/10.1111/ipd.12067

Elhennawy, K., Reda, S., Finke, C., Graul-Neumann, L., Jost-Brinkmann, P.-G., \& Bartzela, T. (2017). Oral manifestations, dental management, and a rare homozygous mutation of the PRDM12 gene in a boy with hereditary sensory and autonomic neuropathy type VIII: a case report and review of the literature. Journal of Medical Case Reports, 11(1), 233. https://doi.org/10.1186/s13256-017-1387-z

Fantuzzo, J. J., Rogér, J. M., Barroner, M. D., \& Karp, J. M. (2014). Self-mutilation of the lower lip in a child with dystonia secondary to megalencephalic leukoencephalopathy treated with botox injections: a case report. Journal of Oral and Maxillofacial Surgery: Official Journal of the American Association of Oral and Maxillofacial Surgeons, 72(7), 1327.e1-4. https://doi.org/10.1016/j.joms.2014.02.016

Flaitz, C. M., \& Felefli, S. (2000). Complications of an unrecognized cheek biting habit following a dental visit. Pediatric Dentistry, $22(6), 511-512$. Gantha, S. N., Chinta, M., Kanumuri, P. K., \& Birra, C. (2017). Non-suicidal self-injury: an unexplored cause of dental trauma. In BMJ case reports (Vol. 2017). https://doi.org/10.1136/bcr-2017-219901

Gao, L., Guo, H., Ye, N., Bai, Y., Liu, X., Yu, P., Xue, Y., Ma, S., Wei, K., Jin, Y., Wen, L., \& Xuan, K. (2013). Oral and craniofacial manifestations and two novel missense mutations of the NTRK1 gene identified in the patient with congenital insensitivity to pain with anhidrosis. PloS One, 8(6), e66863. https://doi.org/10.1371/journal.pone.0066863

Gaur, N., Meel, R., Anjum, S., \& Singh, P. (2018). Hereditary sensory and autonomic neuropathy in a male child: "The other side of not feeling pain”. BMJ Case Reports, 2018. https://doi.org/10.1136/bcr-2018-226873

Gisbert de la Cuadra, L., Torres, R. J., Beltrán, L. M., Sánchez, A., \& Puig, J. G. (2016a). Development of new forms of self-injurious behavior following total dental extraction in Lesch-Nyhan disease. Nucleosides, Nucleotides and Nucleic Acids, 35(10-12), 524-528. https://doi.org/10.1080/15257770.2016.1184276

Gisbert de la Cuadra, L., Torres, R. J., Beltrán, L. M., Sánchez, A., \& Puig, J. G. (2016b). Development of new forms of self-injurious behavior following total dental extraction in Lesch-Nyhan disease. Nucleosides, Nucleotides \& Nucleic Acids, 35(10-12), 524-528. https://doi.org/10.1080/15257770.2016.1184276

Herrera, D. A., \& Pollock, A. N. (2014). Intentional insertion of a foreign body in the tongue in a patient with polyembolokoilamania. Pediatric Emergency Care, 30(5), 368-369. https://doi.org/10.1097/PEC.0000000000000157

Hildebrand, L. C., Carvalho, A. L., da Rosa, F. M., Martins, M. D., \& Filho, M. S. A. (2011). Functional oral self-mutilation in physically healthy pediatric patients: Case report and analysis of 27 literature cases. International Journal of Pediatric Otorhinolaryngology, $75(6)$, 880-883. https://doi.org/10.1016/j.ijporl.2011.03.020

Hildebrand, L. C., Carvalho, A. L., da Rosa, F. M., Martins, M. D., \& Sant'Ana Filho, M. (2011). Functional oral self-mutilation in physically healthy pediatric patients: case report and analysis of 27 literature cases. International Journal of Pediatric Otorhinolaryngology, 75(6), 880-883. https://doi.org/10.1016/j.ijporl.2011.03.020

Kwon, I. J., Kim, S. M., Park, H. K., Myoung, H., Lee, J. H., \& Lee, S. K. (2015). Successful treatment of self-inflicted tongue trauma patient using a special oral appliance. International Journal of Pediatric Otorhinolaryngology, 79(11), 1938-1941. https://doi.org/10.1016/j.ijporl.2015.08.013 
Labib, S., Adnane Berdai, M., Abourazzak, S., Hida, M., \& Harandou, M. (2011). Congenital insensitivity to pain with anhydrosis: report of a family case. The Pan African Medical Journal, 9, 33. https://doi.org/10.4314/pamj.v9i1.71209

Limeres, J., Feijoo, J. F., Baluja, F., Seoane, J. M., Diniz, M., \& Diz, P. (2013). Oral self-injury. An update. Dental Traumatology, 29(1), 8-14. https://doi.org/10.1111/j.1600-9657.2012.01121.x

Malaga, E. G., Aguilera, E. M. M., Eaton, C., \& Ameerally, P. (2016). Management of Self-Harm Injuries in the Maxillofacial Region: A Report of 2 Cases and Review of the Literature. Journal of Oral and Maxillofacial Surgery: Official Journal of the American Association of Oral and Maxillofacial Surgeons, 74(6), 1198.e1-9. https://doi.org/10.1016/j.joms.2016.02.018

Medina, A. C., Sogbe, R., Gómez-Rey, A. M., \& Mata, M. (2003). Factitial oral lesions in an autistic paediatric patient. International Journal of Paediatric Dentistry, 13(2), 130-137. https://doi.org/10.1046/j.1365-263x.2003.00440.x

Mistry, L. N., Hugar, S. M., Patil, V. H., \& Patel, P. (2017). Successful Management of the Masochistic Habit in a Child with Santovuori-Haltia-Hagberg Disease (Infantile Neuronal Ceriod Lipofuscinoses). In Journal of clinical and diagnostic research: JCDR (Vol. 11, Issue 1, pp. ZD47-ZD49). https://doi.org/10.7860/JCDR/2017/24120.9286

Mistry, P., Moles, D. R., O\&\#039;Neill, J., \& Noar, J. (2010). The occlusal effects of digit sucking habits amongst school children in Northamptonshire (UK). J Orthod, 37(2), 87-92. http://dx.doi.org/10.1179/14653121042939

Moher D, Liberati A, Tetzlaff J, A. D. (2009). Preferred Reporting Items for Systematic Reviews and Meta-Analyses: The PRISMA Statement. Plos Mend, 6, doi:10.1371/journal.pmed1000097.

Mowafy, Y. N., Wahba, N. A., \& Sharaf, A. A. (2017a). Joubert Plus Syndrome with Self-Mutilation: A Case report. The Journal of Clinical Pediatric Dentistry, 41(1), 66-69. https://doi.org/10.17796/1053-4628-41.1.66

Mowafy, Y. N., Wahba, N. A., \& Sharaf, A. A. (2017b). Joubert plus syndrome with self-mutilation: A case report. Journal of Clinical Pediatric Dentistry, 41(1), 66-69. https://doi.org/10.17796/1053-4628-41.1.66

MURSHID, E. (2005). Oral health status, dental needs, habits and behavioral attitude towards dental treatment of a group of autistic children in Riyadh, Saudi Arabia. Saud Dent J, 17(1013-9052), 132-139.

Navya, M. K., Pramod, G. V, Sujatha, G. P., \& Ashok, L. (2019). Congenital insensitivity to pain in a 1 -year-old boy. In Journal of the Indian Society of Pedodontics and Preventive Dentistry (Vol. 37, Issue 3, pp. 308-310). https://doi.org/10.4103/JISPPD.JISPPD_340_18

Olczak-Kowalczyk, D., Korporowicz, E., Gozdowski, D., Lecka-Ambroziak, A., \& Szalecki, M. (2019). Oral findings in children and adolescents with PraderWilli syndrome. Clin Oral Investig, 23(3), 1331-1339. https://dx.doi.org/10.1007/s00784-018-2559-y

Olczak-Kowalczyk, D., Witt, A., Gozdowski, D., \& Ginalska-Malinowska, M. (2011). Oral mucosa in children with Prader-Willi syndrome. Journal of Oral Pathology \& Medicine: Official Publication of the International Association of Oral Pathologists and the American Academy of Oral Pathology, 40(10), 778-784. https://doi.org/10.1111/j.1600-0714.2011.01034.x

Panek, H., Nawrot, P., Mazan, M., Bielicka, B., Sumislawska, M., \& Pomianowski, R. (2012). Coincidence and awareness of oral parafunctions in college students. Community Dent Health, 29(1), 74-77. https://pesquisa.bvsalud.org/portal/resource/pt/mdl-22482254

Pereira A.S. et al. (2018). Metodologia da pesquisa científica. [e-book]. Santa Maria. Ed. UAB/NTE/UFSM. Recuperado de: em: https://repositorio.ufsm.br/bitstream/handle/1/15824/Lic_Computacao_Metodologia-Pesquisa-Cientifica.pdf?sequence=1

Rangeeth, B. N., Moses, J., \& Reddy, N. V. (2011). Self-injurious behavior and foreign body entrapment in the root canal of a mandibular lateral incisor. $J$ Indian Soc Pedod Prev Dent, 29 (6 Suppl 2), S95-8. https://dx.doi.org/10.4103/0970-4388.90754

Rodd, H. D. (1995). Self-inflicted gingival injury in a young girl. British Dental Journal, 178(1), 28-30. https://doi.org/10.1038/sj.bdj.4808639

Romero, M., Vicente, A., \& Bravo, L. A. (2005). Prevention of habitual cheek biting: a case report. Special Care in Dentistry : Official Publication of the American Association of Hospital Dentists, the Academy of Dentistry for the Handicapped, and the American Society for Geriatric Dentistry, 25(4), 214-216. https://doi.org/10.1111/j.1754-4505.2005.tb01652.x

Romero Maroto, M., Ruiz Duque, C., Vincent, G., Garcia Recuero, I., \& Romance, A. (2014). Management of oral lesions in Lesch-Nyham syndrome. J Clin Pediatr Dent, 38(3), 247-249. https://pesquisa.bvsalud.org/portal/resource/pt/mdl-25095320

Shapira, J., Birenboim, R., Shoshani, M., Abdel-Kader, A., Behar, O., Moskovitz, M., Ben-Attar, Y., Chaushu, S., \& Becker, A. (2016). Overcoming the oral aspects of -self-mutilation: description of a method. Special Care in Dentistry: Official Publication of the American Association of Hospital Dentists, the Academy of Dentistry for the Handicapped, and the American Society for Geriatric Dentistry, 36(5), 282-287. https://doi.org/10.1111/scd.12181

Siragusa, M., Ferri, R., Russo, R., Lentini, M., \& Schepis, C. (2013). Self-inflicted lesions of the mouth and lips in mentally retarded young subjects. European Journal of Dermatology : EJD, 23(6), 843-848. https://doi.org/10.1684/ejd.2013.2172

Soussou, R., Cheung, W. S., \& Campbell, K. M. (2019). Congenital insensitivity to pain with anhidrosis: A case with self-inflicted oral ulcerations. Journal of Dentistry for Children, 86(2), 109-112.

Souza MT, Silva MD, C. R. (2010). Revisão integrativa: o que é e como fazer. Einstein, 8(1), 102-106. https://doi.org/10.1590/S1679-45082010RW1134 Stewart, D. J., \& Kernohan, D. C. (1972). Self-inflicted gingival injuries. Gingivitis artefacta, factitial gingivitis. The Dental Practitioner and Dental Record, $22(11), 418-426$

Stewart, D. J., \& Kernohan, D. C. (1973). Traumatic gingival recession in infants. The result of a dummy sucking habit. British Dental Journal, 135(4), 157158. https://doi.org/10.1038/sj.bdj.4803058 
Research, Society and Development, v. 10, n. 2, e29710212493, 2021

(CC BY 4.0) | ISSN 2525-3409 | DOI: http://dx.doi.org/10.33448/rsd-v10i2.12493

Subbaiah R, Thomas B, M. V. (2015). case series Self-inflicted traumatic injuries of the gingiva- A case series. J Int Oral Health, 2 (January 2010$)$, 43-49. Suhaib, F., Saeed, A., Gul, H., \& Kaleem, M. (2017). Oral assessment of children with autism spectrum disorder in Rawalpindi, Pakistan. Autism, 23(1), 8186. https://doi.org/10.1177/1362361317730299

Suhaib, F., Saeed, A., Gul, H., \& Kaleem, M. (2019). Oral assessment of children with autism spectrum disorder in Rawalpindi, Pakistan. Autism : The International Journal of Research and Practice, 23(1), 81-86. https://doi.org/10.1177/1362361317730299

Tate, B. G., \& Baroff, G. S. (1966). Aversive control of self-injurious behavior in a psychotic boy. Behaviour Research and Therapy, 4(4), 281-287. https://doi.org/10.1016/0005-7967(66)90024-6

Topouzelis, N., Kotsiomiti, E., \& Arhakis, A. (2010). An alternative impression technique for individuals with special care needs. Special Care in Dentistry: Official Publication of the American Association of Hospital Dentists, the Academy of Dentistry for the Handicapped, and the American Society for Geriatric Dentistry, 30(6), 266-270. https://doi.org/10.1111/j.1754-4505.2010.00165.x

Wadenya, R., Pinto, A., \& Lindemeyer, R. (2011). Oral factitious injury in a child diagnosed with Kabuki syndrome. Compendium of Continuing Education in Dentistry (Jamesburg, N.J. : 1995), 32(1), E1-3.

Williams, A. C. (2015). Autoextraction of twelve permanent teeth in a child with autistic spectrum disorder. International Journal of Paediatric Dentistry, 26(2), 157-159. https://doi.org/10.1111/ipd.12161

Williamson, A., D’Este, C., Clapham, K., Redman, S., Manton, T., Eades, S., Schuster, L., \& Raphael, B. (2016). What are the factors associated with good mental health among Aboriginal children in urban New South Wales, Australia? Phase I findings from the Study of Environment on Aboriginal Resilience and Child Health (SEARCH). BMJ Open, 6(7), e011182. https://doi.org/10.1136/bmjopen-2016-011182 